\title{
Upper Airway Evaluation in Patients with Obstructive Sleep Apnea
}

\author{
Seung-No Hong, $\mathrm{MD}^{1,2}$, Tae-Bin Won, MD, $\mathrm{PhD}^{3}$, Jeong-Whun Kim, MD, PhD², \\ Chul Hee Lee, MD, PhD², Chae-Seo Rhee, MD, PhD 2,3 \\ 'Department of Otorhinolaryngology, Korea University College of Medicine, Korea University Ansan Hospital, Ansan, Korea \\ 2Department of Otorhinolaryngology, Seoul National University College of Medicine, Seoul National University Bundang Hospital, Seongnam, Korea \\ ${ }^{3}$ Department of Otorhinolaryngology, Seoul National University College of Medicine, Seoul National University Hospital, Seoul, Korea
}

\begin{abstract}
Obstructive sleep apnea (OSA) is a widespread disease of substantial social burden. As various surgical procedures are now developed to alter the offending anatomic abnormalities, identification of the exact problematic site with application of the most appropriate treatment including surgical procedure is essential for effective surgical treatment of OSA. To date, many techniques are available for the physician to assess and analyze the upper airway obstruction including radiographic cephalometry, sleep video fluoroscopy, computed tomography imaging, sleep magnetic resonance imaging, drug induced sleep endoscopy, multi-channel pressure measurements, acoustic reflectometry, and basic physical examinations. However there are still some controversies concerning the effectiveness and morbidity of each technique. Therefore, sleep medicine doctors should understand the characteristics of each tool with the pathophysiology of OSA. Herein, we review the clinical methods to evaluate the upper airway in terms of disease severity and treatment selection.
\end{abstract}

Sleep Med Res 2016;7(1):1-9

Key Words Obstructive sleep apnea, Airway obstruction, Diagnosis.

Received: October 30, 2015

Revised: February 15, 2016

Accepted: February 29, 2016

Correspondence

Chae-Seo Rhee, MD, PhD

Department of Otorhinolaryngology,

Seoul National University College of Medicine,

Seoul National University Bundang Hospital,

82 Gumi-ro 173beon-gil, Bundang-gu,

Seongnam 13620, Korea

Tel +82-31-787-7401

Fax +82-31-787-4057

E-mail_csrhee@snu.ac.kr

\section{INTRODUCTION}

Obstructive sleep apnea (OSA) is an increasingly common disease with pathophysiology based on the interaction of multiple factors. Continuous positive airway pressure (CPAP) is considered as the treatment of choice for OSA syndrome, but poor patient acceptance and compliance remain problematic. Surgical procedures are developed to alter the offending anatomic abnormalities responsible for OSA. Identification of the offending anatomic site with application of the most appropriate surgical procedure is essential for effective surgical treatment of OSA. The standard for the diagnosis of OSA is polysomnography (PSG). Although PSG helps to identify individuals who have OSA and guides medical management, it does not identify the site of obstruction or predict surgical results.

Various radiologic and diagnostic studies are developed to evaluate the obstruction site. These tools permit not only the diagnosis of OSA but also the understanding of its pathophysiology, identification of subjects with increased OSA risk, and selection of the more appropriate treatment modality. On the other hand, current outcomes of surgery for OSA are still poor. This may be due to the fact that OSA itself is not solely an anatomical problem and surgery that is designed to achieve only an anatomical modification is thus insufficient. The failure to identify the anatomical regions of upper airway obstruction and specific structures [soft palate (SP), tonsils, lateral pharyngeal walls, tongue base, and/or epiglottis] contributing to this obstruction are other factors. Therefore, identifying the anatomical locations and the pattern of obstruction and integrating this anatomy into proper surgical technique are essential for improving effectiveness and minimizing morbidity. The Ideal measurement characteristics of evaluation methods are accurateness, high repeatability and low inter-rater variability. Moreover, the method should be simple, practical, less expensive and noninvasive without radiation exposure. An explanation of methods for upper airway evaluation and their advantages 
and disadvantages are included in the following.

\section{PHYSICAL EXAMINATIONS WITH CLINICAL SCORES}

Physical examination including an endoscopic examination of the upper airway (nasal cavity, nasopharynx, oropharynx, hypopharynx, or larynx) during wakefulness still constitutes the basis of every airway evaluation in snorers. Physical and endoscopic evaluation of the upper airway has a critical role in the decision of patient management and treatment. It has several significant limitations such as the subjective assessment and the variability of the nomenclature of the clinical findings. However clinical and endoscopic evaluation is essential in the preoperative assessment or treatment decision with mandibular advancement devices (MADs).

Modified Friedman staging is the most commonly used evaluation system to predict the surgical outcome of OSA patients. ${ }^{1,2}$ They developed an evaluation system by assessing the size of tonsil, the position of the SP, the volume of the tongue and the body mass index (BMI) which makes a 4 degree clinical staging system. The success rate of uvulopalatopharyngoplasty (UPPP) with tonsillectomy was $80 \%$ when patients had large tonsils, visible posterior pharyngeal wall and a BMI below $40 \mathrm{~kg} / \mathrm{m}^{2}$. This staging system is much better than the conventional OSA severity score in predicting the success rate of surgery. However, whether using these staging systems is better than simple physical examinations by an experienced physician remains to be determined. Our team has studied the relationship between severity of apnea and anthropometric oropharyngeal measurements by physical examination in 22 patients with snoring and OSA. The anthropometric measurements, show that the horizontal width of the uvula is significantly correlated with respiratory disturbance index (RDI) and lowest $\mathrm{SaO}_{2}$ (Fig. 1). In addition, the results of the study indicate that patients with broader uvula may have severer sleep apnea and that anthropometric oropharyngeal measurements may give additional information to polysomnographic findings for selecting surgical candidates. $^{3}$

\section{CEPHALOMETRIC ANALYSIS}

Currently, lateral cephalometric radiography is one of the most popular OSA evaluation methods. The use of modern cephalometric radiography to evaluate the level of obstruction site in OSA was proposed by Riley et al. ${ }^{4}$ in 1983 . Though it has a limitation of studying a three-dimensional object with a twodimensional picture, it is an inexpensive, quick and simple tool for assessing the pharyngeal airway. Simple radiographic imaging (X-ray) of the maxilla, mandible and the upper airway are

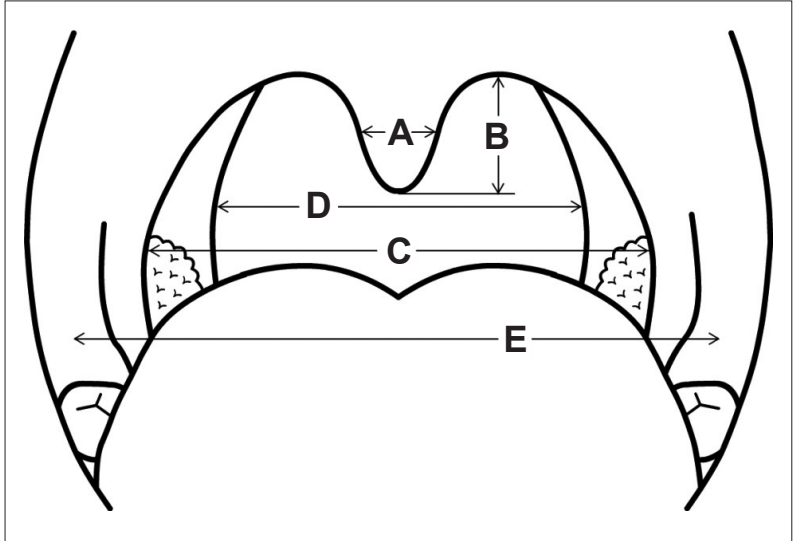

Fig. 1. Schematic drawing of anthropometric oropharyngeal measurements. The width of the uvula at mid-point (A), the length of the uvula (B), the longest distance between the anterior pillars (C), the longest distance between the posterior pillars (D), and the distance between the retro molar raphes $(E)$ are depicted.

taken while the patient stands upright with one's head in the cephalostat which is on the horizontal Frankfurt plane, at the end of expiration. Comparison between OSA patients and healthy controls are frequently reported in many literatures. The reported differences of cephalometric characters between OSA patients and healthy controls include an elongated SP, a narrow palatal airway widths, an increased thickness of the SP, a differences in craniofacial score which is the sums of quartile points for distance from sella to nasion and hyoid bone to mandible, an extended pharyngeal length, a retroposition of the mandible, or the maxilla, a micrognathia, an increased mid-facial height, and an alterations in hyoid bone position in OSA patients. ${ }^{5,6}$

In correlation with disease severity aberrations in craniofacial morphology (the above mentioned characteristics) are more definite in patients with severe OSA. However the surgical predictive value of cephalometry for UPPP remains at least questionable. Our department demonstrated that the simple measurement of mouth-opening angle could outstandingly predict surgical outcome. Cephalometry and multivariate analysis on 69 of 120 consecutive patients with OSA who underwent UPPP shows that an increased angle of mouth opening during sleep is the only significant predictor for surgical failure $(\mathrm{p}<0.001){ }^{7}$

Lateral cephalometry is also indicated when oral MADs are considered. By evaluating specific anatomical parameters, cephalometric analysis can be helpful in predicting the treatment outcome and is also widely performed to assess the long term side effects of oral appliances including occlusion and dental problem. ${ }^{8,9}$

\section{SLEEP VIDEO FLUOROSCOPY}

Sleep video fluoroscopy (SVF) is a readily available technique to assess dynamic airway anatomy and sites of obstruction in 
OSA patients. Advantages of SVF include direct observation of obstructive sites during episodes of apnea, and availability of fluoroscopy in most hospitals. Drawbacks are high radiation dose, superimposition of structures, the possible need for sedation to attain sleep during the procedure, evaluating only a few apneic events, only two dimensional and highly specialized interpretation, therefore limiting its use. However according to our study on 374 consecutive OSA patients, the inter-rater reliability of SVF evaluation for OSA is excellent and its analysis results performed by a less-experienced sleep surgeon were quite comparable to those by experienced sleep surgeons, suggesting its short learning curve. ${ }^{10}$

In evaluation of upper airway with SVF, Walsh et al. ${ }^{11}$ show that the site of obstruction and site of initiation of obstruction were variable among a group of 40 patients, that hypopharyngeal collapse correlates well with inferior displacement of the hyoid bone, and that surgical success with UPPP improves from $42 \%$ to $67 \%$ by selecting patients with obstruction initiation in the oropharynx. From our experience of a large cohort study (922 OSA patients who underwent both PSG and SVF) even if multiplicity of obstruction pattern is most commonly associated with severe OSA, almost one third (32.4\%) of mild OSA patients also show multiple anatomic structure obstruction (Table 1). Therefore, a precise evaluation for multiplicity of obstruction levels should precede the decision of a treatment plan, regardless of disease severity. ${ }^{12}$ In addition, SVF not only shows the dynamic airway alterations in patients with OSA but also the multiple effects of MAD on the dimension and configuration of the upper airway. From 68 patients who were prescribed with the MAD for OSA, we determine that MAD extends the retropalatal and retrolingual spaces and shortens the length of the SP and decreases the angle of mouth opening, leading to an improvement of OSA. ${ }^{13}$

\section{COMPUTED TOMOGRAPHY IMAGING}

CT scanning shows a precise measurement of bony framework and cross-sectional area of the airway at different levels and can be reconstructed in three dimensional images. The volumetric assessment and improved soft tissue contrast makes the difference with radiographic cephalometry. Most physicians use CT imaging during wakefulness. But quick scanning times and relatively silent scanning conditions even enables a dynamic evaluation of the airway during a whole respiratory cycle and also investigations during natural sleep or drug induced hypnotic state.

In relation to disease severity, the cross-sectional areas of the OSA patients are considerably narrower. In contrast, compared to controls, OSA patients show a greater retropalatal tissue area. Dynamic CT study shows a narrower cross-sectional area and a larger SP in severely affected patients compared to patients with only mild-to-moderate OSA. ${ }^{14}$ The treatment response of oral appliances and surgical intervention can be assessed with the help of upper airway CT imaging. In 23 OSA patients, UPPP shows double the upper airway cross-sectional and OSA patients with smaller upper airway volumes show a greater response to UPPP. ${ }^{15}$ The advantages of CT scanning include the ability to scan the entire airway, providing accurate measurement of the airway, permitting cross-sectional as well as threedimensional evaluation, and the noninvasive nature of the technique. The disadvantages of CT scanning are relatively costly, the inability to image the entire pharyngeal airway in a single plane, the ability to record only a short period of time, and the health risk of radiation exposure. To date, using cine CT or ultra-fast CT allows physician to obtain multiple images with less radiation. However the limitation of CT in comparison with MR, particularly its poor resolution in detection of airway fat cause less frequent use of CT in OSA evaluation. ${ }^{16}$

Table 1. Anatomic structures associated with obstruction during desaturation sleep events

\begin{tabular}{|c|c|c|c|c|}
\hline & Mild OSA (\%) & Moderate OSA (\%) & Severe OSA (\%) & Total (\%) \\
\hline SP & $76(43.2)$ & $132(42.9)$ & $139(31.7)$ & $347(37.6)$ \\
\hline $\mathrm{TB}$ & $24(13.6)$ & $45(14.6)$ & $34(7.8)$ & $103(11.2)$ \\
\hline $\mathrm{SP}+\mathrm{TB}$ & $47(26.7)$ & $102(33.1)$ & $198(45.2)$ & $347(37.6)$ \\
\hline Epi & $7(4.0)$ & $3(1.0)$ & $9(2.1)$ & $19(2.1)$ \\
\hline SP + Epi & $7(4.0)$ & $12(3.9)$ & $18(4.1)$ & $37(4.0)$ \\
\hline TB + Epi & $2(1.1)$ & $4(1.3)$ & $10(2.3)$ & $16(1.7)$ \\
\hline Tonsil & $12(6.8)$ & $8(2.6)$ & $24(5.5)$ & $44(4.8)$ \\
\hline $\mathrm{TB}+$ tonsil & $1(0.6)$ & $2(0.6)$ & $6(1.4)$ & $9(1.0)$ \\
\hline Single structure & $119(67.6)$ & $188(61.0)$ & $206(47.0)$ & $513(55.6)$ \\
\hline Multiple structures & $57(32.4)$ & $120(39.0)$ & $232(53.0)$ & $409(44.4)$ \\
\hline Total & $176(100)$ & $308(100)$ & $438(100)$ & $922(100)$ \\
\hline
\end{tabular}

OSA: obstructive sleep apnea, SP: soft palate, TB: tongue base, Epi: epiglottis. 


\section{MAGNETIC RESONANCE IMAGING}

MRI was first applied for the evaluation of OSA on 1989. Significantly, clear soft tissue resolution, three-dimensional evaluation of structures with no radiation exposure is the unique advantage of MRI. Especially, in the upper airway evaluation of children, the absence of radiation hazard makes MRI the imaging of choice in OSA. With newer technologies, the dynamic airway can be evaluated with rapid image acquisition; multiple images per second. From our experience, sleep MRI could be a valuable method for evaluating dynamic obstruction during
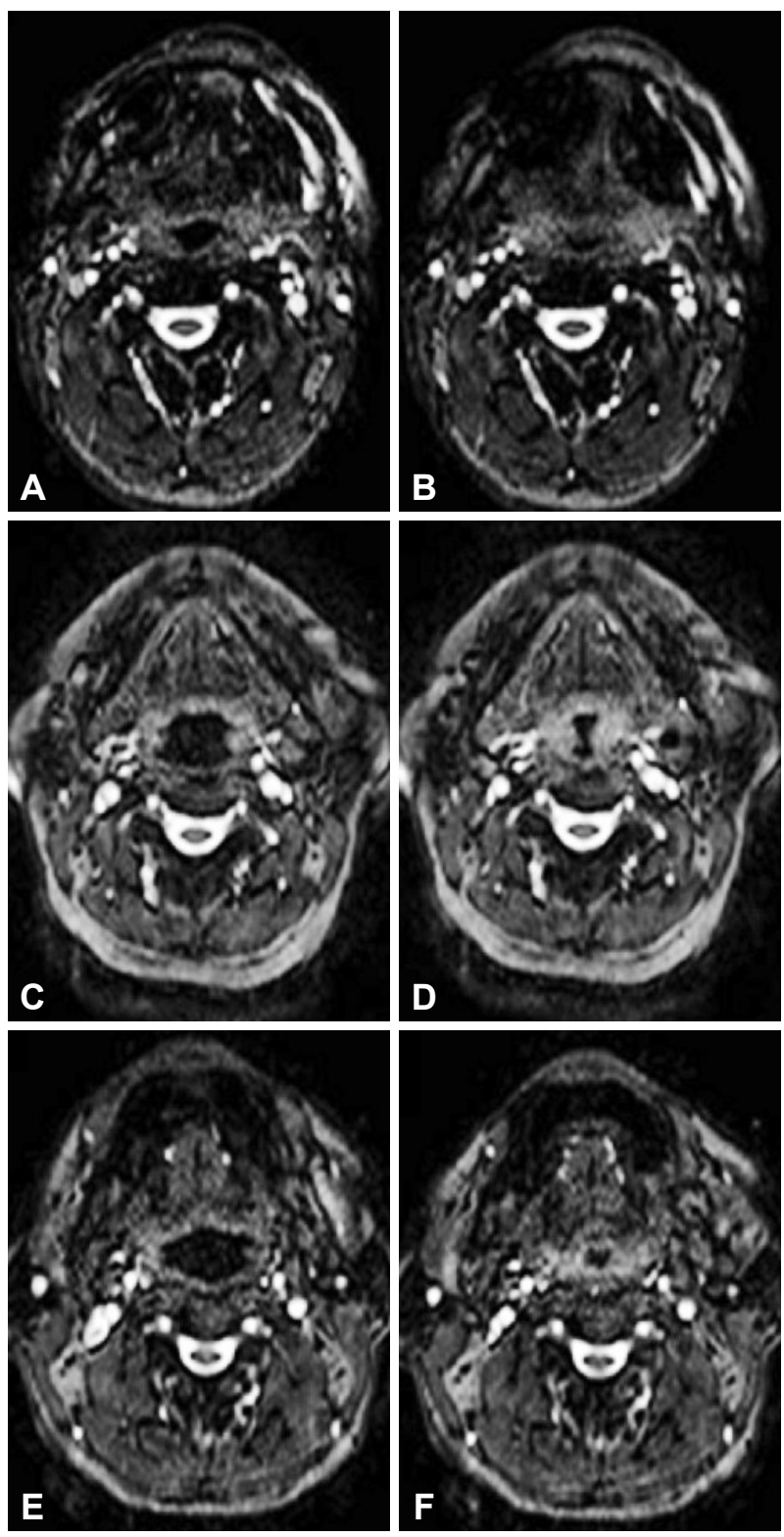

Fig. 2. Axial magnetic resonance images at the retro lingual level which represents the anteroposterior collapse (A and $B)$, the transverse collapse ( $C$ and $D)$, and the circumferential collapse $(E$ and $F$ ). sleep and may be helpful in selecting the appropriate treatment approaches. By analyzing 35 snoring subjects with sleep MRI, we find that airway collapse occurs in the retropalatal area in 13 (37.1\%) of 35 subjects and in both the retropalatal and retrolingual regions in 20 subjects (57.1\%) (Fig. 2). Supine RDI, minimal oxygen saturation during sleep and age are significantly different between anteroposterior, transverse, and circumferential collapses in axial images of MR. ${ }^{17}$

The implementation of MRI in evaluating OSA has been difficult because of the nature of the examination. Difficulties arise with regards to patient comfort, concurrent sleep evaluation, scanner noise with possible requirement of sedation, and examination expense. Unfortunately, in diagnosis of disease severity, most studies fail to distinguish OSA from control patients. Nevertheless in terms of evaluating the surgical outcome, MRI has a significant role. In radiofrequency operation, MRI is performed to access the prompt post-operative effects on tissue structures including the tongue base and the SP..$^{18}$ Interestingly, Huang et al. ${ }^{19}$ report a computational model of the human upper airway based on signal averaging of MRI. By finite element method, this model enables simulation of various surgical interventions and shows the postoperative movement and obstruction pattern, facilitating the development and improvement of the surgical and non-surgical modalities. There are still some limitations in evaluating the upper airway with MRI. First, performing MRI during natural sleep is technically difficult and assessments during awaken state or drug induced sleep are, in some point, non-physiologic or do not imply the real clinical conditions. Moreover, the rather short exam time and limited sleep position can provide false information of the patient.

\section{MULLER MANEUVER}

Before discussing video endoscopy during sedation, endoscopic examination technique during awake state should be mentioned such as the Muller maneuver. Mueller maneuver is described as follows. While observing the pharyngeal space with a flexible fiber optic scope, the awaken patient is sitting or lying and inspiring maximally with nose and mouth closed. The level of the supraglottis, the uvula tip and the nasopharynx is assessed via the endoscope. In predicting the airway obstruction during sleep, unfortunately several studies report that Mueller maneuver is not efficient to reveal the obstruction site during sleep. Moreover, some different sites of obstruction cannot be detected by the Muller maneuver in awake status. These results are demonstrated in a comparison study with video endoscopy, ${ }^{20,21}$ multichannel pressure recordings ${ }^{22}$ and dynamic MRI during sleep. ${ }^{23}$

There is poor evidence for predicting the surgical outcome. In case of obstruction found at the hypopharynx level, surgeon should exclude patients from UPPP, which indirectly increases 
the success rate. Many studies document that Mueller maneuver is less effective in predicting the surgical outcome after UPPP. ${ }^{24-26}$ Though the reliability of the Mueller maneuver is in-

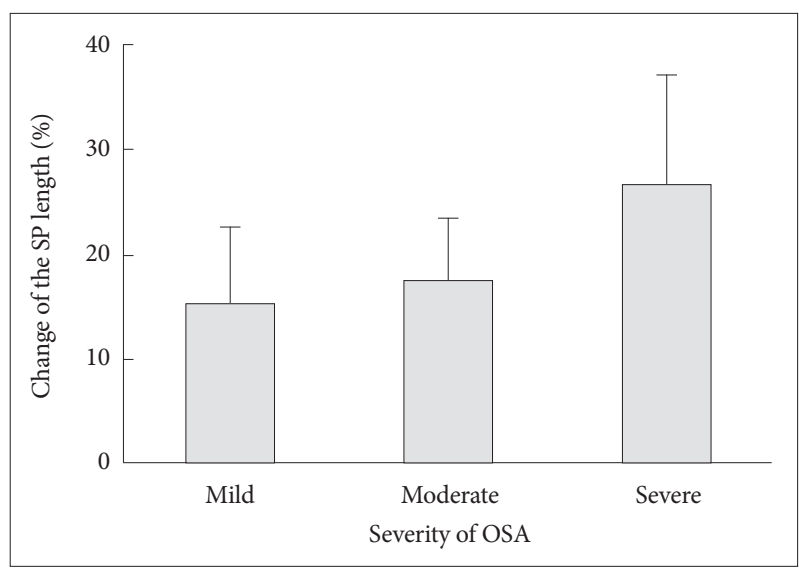

Fig. 3. Change in the soft palate (SP) length according to the severity of apnea/hypopnea index (AHI). The change of the SP length is greater in patients with severe $\mathrm{AHI}$ than in those with mild or moderate AHI. Error bars indicate standard deviations. OSA: obstructive sleep apnea.
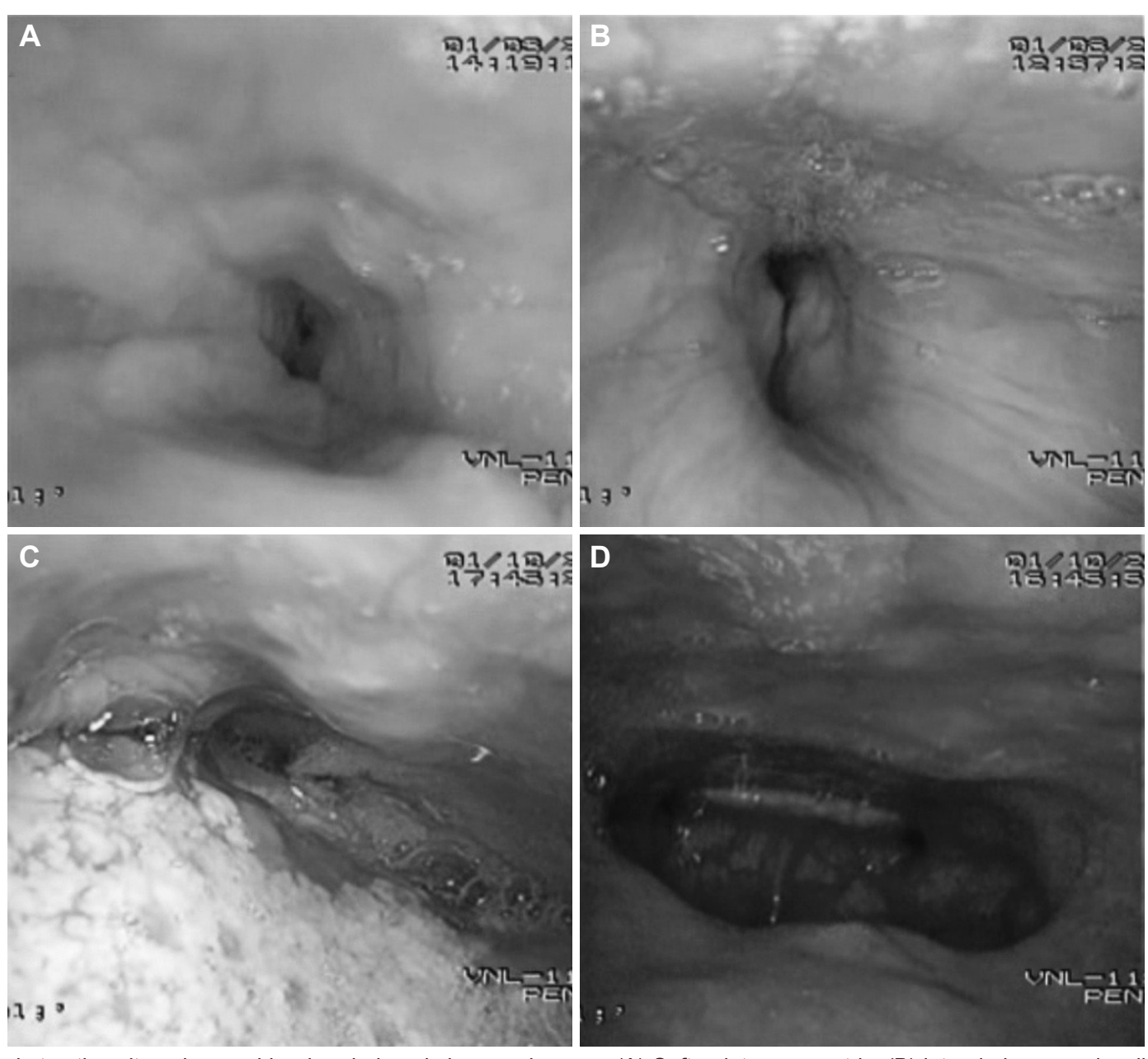

Fig. 4. The obstructive sites observed by drug induced sleep endoscopy. (A) Soft palate: concentric, (B) lateral pharyngeal wall, (C) tongue base, (D) epiglottis. sufficient, the Mueller maneuver is still a simple and safe examination without causing relevant strain on the patient. Highly subjective, hard to reproduce results can be overcome by the performance of computer-aided assessment modalities. In 63 patients with snoring of sleep apnea who underwent videofluoroscopy, we show that the SP is considerably elongated and angulated ( $\mathrm{p}<0.01$ for SP length; $\mathrm{p}=0.03$ for SP angle) in patients with OSA even in wakefulness (Fig. 3). We show that it could be an easy method to assess the SP changes and may be a useful tool to distinguish OSA from simple snoring in short examination time. ${ }^{27}$ Nevertheless, the results of the Mueller maneuver cannot be transferred to natural sleep. And Mueller maneuver does not facilitate patient selection for the varying surgical interventions used in OSA patients.

\section{DRUG INDUCED SLEEP ENDOSCOPY}

Spontaneous sleep endoscopy is an actual upper airway evaluation tool for selecting patients and treatment methods. More information can be obtained when the sleep endoscopy is per- 
formed with full overnight PSG datas. Evaluation of the airway by different sleep stages is possible without the side effects of sedating drugs, which makes this method superior to video endoscopy under sedation. However, spontaneous sleep video endoscopy is seldom done because it needs nightly assessments and gives extra strain on the doctor as well as the patient.

Drug induced sleep endoscopy provides the information of obstruction site and snoring mechanism in patients with OSA. It is reported that most of the factors including the scope or the sedatives do not affect the obstruction pattern or the breathing dynamics except for the longest duration of apnea and duration of REM sleep. ${ }^{28}$ On the other hand, an early study shows that $18 \%$ of previous snorers do not snore and $45 \%$ of previous non-snorers snore during drug induced sleep. ${ }^{29}$ However, computer-controlled infusion system using propofol causes 100 percent of snorers to snore, while 100 percent of non-snorers do not snore. ${ }^{30}$ The form of upper airway obstruction and snoring, which is assessed during video endoscopy under sedation are multiform. There are several obstruction types and levels, which include circular, antero-posterior and latero-lateral at the level of the SP, the tonsils, the tongue base and the epiglottis (Fig. 4). In terms of treatment selection, drug induced sleep endoscopy does give significant information. In a study on 324 OSA patients in wakefulness performing Mueller maneuver and also in propofol induced state, drug induced sleep endoscopy shows more obstruction at SP and tongue base compared to Mueller maneuver during awaken status. ${ }^{31}$ In another study, four of 27 patients canceled their plan for UPPP after performing videoendoscopy under sedation due to a tongue base collapse. Unfortunately there was no surgical success rate gain on performing drug induced sleep endoscopy, as compared to conventional methods in that study. ${ }^{32}$

In summary drug induced sleep endoscopy, although during a short time of induced sleep and restricted in supine position, can recreate snoring and upper airway obstruction. While it has some limitation such as different character of snoring sound and short examination period, the severity of OSA is comparable to spontaneous sleep. The classification of endoscopic findings can be simplified to isolated obstruction at the velum, oropharyngeal lateral walls, tongue base, epiglottis or the combination of these. ${ }^{33}$ Drug induced sleep endoscopy is able to change the surgical plan for OSA. However, this procedure is still not available to change in the surgical outcome of snoring and sleep apnea. Another pitfall of drug induced sleep endoscopy is the subjectivity in evaluation, although it may be overcome by a clear defined procedure protocol.

\section{MULTI-CHANNEL PRESSURE MEASUREMENTS}

With channel catheters, it is possible to measure the change of pressure in airway at sleep apnea episodes. Multiple pressure measuring points can be used to check the change of inspiratory pressures including the nasopharyngeal, oropharyngeal, hypopharygeal space and the esophagus. Several conditions are needed to provide good results from this examination. First the catheter must not disturb the sleep or breathing. Second, the measuring point should not be altered after the examination starts since monitoring of the location is difficult. It is demonstrated that a precise evaluation of the obstruction level of the airway is crucial before performing a surgical intervention. ${ }^{34}$ According to our study on 45 patients evaluated by PSG, multisensor manometer during the full night, patients with OSA showing the same anatomical stage, and same Friedman tongue position (FTP) have varying degrees of retroglossal obstruction, stressing the heterogeneous nature of obstruction within a group of anatomically based stage. Thus, FTP correlates with retroglossal obstruction and may be used to select patients in need of retroglossal modification of the airway (Table 2). ${ }^{35}$ If the diameter of the catheter does not exceed $2 \mathrm{~mm}$, it does not affect the patients sleep quality and airway obstructions. Respiratory events are detected by the resulting pressure curves. It is sufficiently accurate to locate the measuring points while inspecting the pharynx for the evaluation of the palatal airway segment.

The pressure catheters have several benefits. First, the evaluation can be performed during natural sleep without disturbing its pattern. Second, long time assessment is possible, which is rather unique among other evaluation methods. This provides high correlation with PSG. However, whether the pressure catheter reflects the airway obstruction or just the pressure difference is still unclear. Moreover, the actual related structure is not depicted, and the detecting probe itself can easily be disturbed producing artifacts. Lastly, this method cannot evaluate multi-obstruction level instead detecting the lowest level of airway obstruction.

Table 2. Percentage of retroglossal obstruction according to the tongue position

\begin{tabular}{ccccc}
\hline Tongue position & Retroglossal obstruction (\%) & BMI & AHI & SpO \\
\hline 1 & $22.1 \pm 30.3$ & $27.4 \pm 3.1$ & $37.7 \pm 23.7$ & $82.0 \pm 5.1$ \\
2 & $46.3 \pm 29.5$ & $27.4 \pm 3.4$ & $45.5 \pm 28.7$ & $78.0 \pm 5.8$ \\
3 & $53.5 \pm 34.8^{*}$ & $27.5 \pm 2.9$ & $35.1 \pm 21.7$ & $77.9 \pm 9.5$ \\
\hline
\end{tabular}

${ }^{*}$ p value $<0.05$.

BMI: body mass index, AHI: apnea hypopnea index. 


\section{ACOUSTIC REFLECTOMETRY, ACOUSTIC ANALYSIS}

Acoustic reflectometry is based on the principle that sound reflects differently by the cross sectional area of the airway space because of the impedance change. This method has several advantages. It is inexpensive, non-invasive, easy to perform, does not disturb sleep, can evaluate multiple apnea events

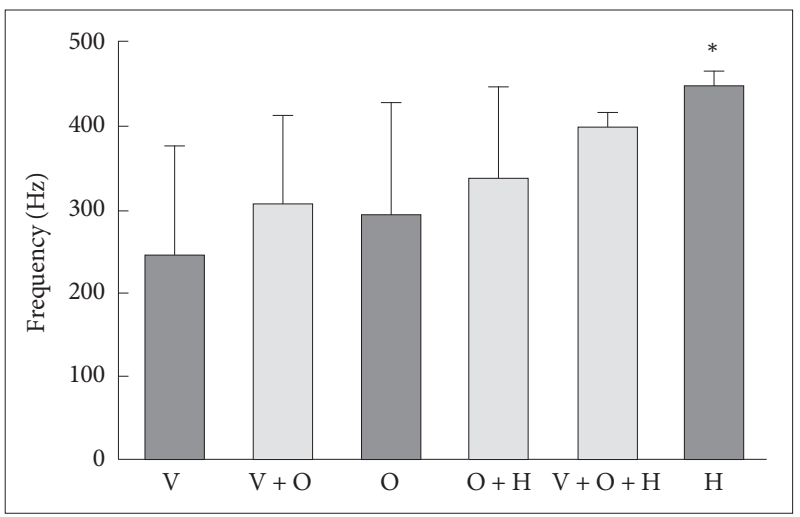

Fig. 5. Mean pitch frequency of snoring sound according to the level of obstruction. Pitch frequency is lowest in the $\mathrm{V}$ obstruction group. Only the $\mathrm{H}$ obstruction group shows higher pitch frequency compared with the velopharyngeal group.* $p<0.05$ compared with $\mathrm{V}$. H: hypopharyngeal, O: oropharyngeal, V: velopharyngeal. with simultaneous recording of PSG without any radiation exposure. However there are certain limitations including its dependency on the patient's position or anatomical structures, the velum or tongue. It is also influenced by other devices such as the mouthpiece or the wave tube. The airway itself changes dynamically during breathing. A previous study shows that the results of acoustic reflectometry are more precise at the level of tongue base than the level of SP. ${ }^{36}$ Studies on acoustic characteristics of snoring sound focus on differentiating simple snorers from patients with OSA. ${ }^{37}$ Further studies have shown that palatal snoring can be distinguished from tongue base snoring by their acoustic features. ${ }^{38}$ Recently, correlation between the snoring sound and the site of obstruction in patients with OSA is reported. ${ }^{39}$ From analysis of 90 patients who underwent simultaneous snoring sound recording during SVF, our team reports that mean value of peak frequency shows significant difference between SP and isolated tongue base or epiglottis obstruction and combined obstruction involving SP and tongue base or epiglottis. Peak frequency of velopharyngeal obstruction showed difference only with hypopharyngeal obstruction (Fig. 5). First formant shows similar results in the structure classification; whereas, velopharyngeal obstruction shows significant difference compared with other levels of obstruction. Other parameters (intensity, jitter, shimmer) do show significance according to site of obstruction. ${ }^{40}$ Therefore snoring sound analysis can complement determination of the site of obstruction in

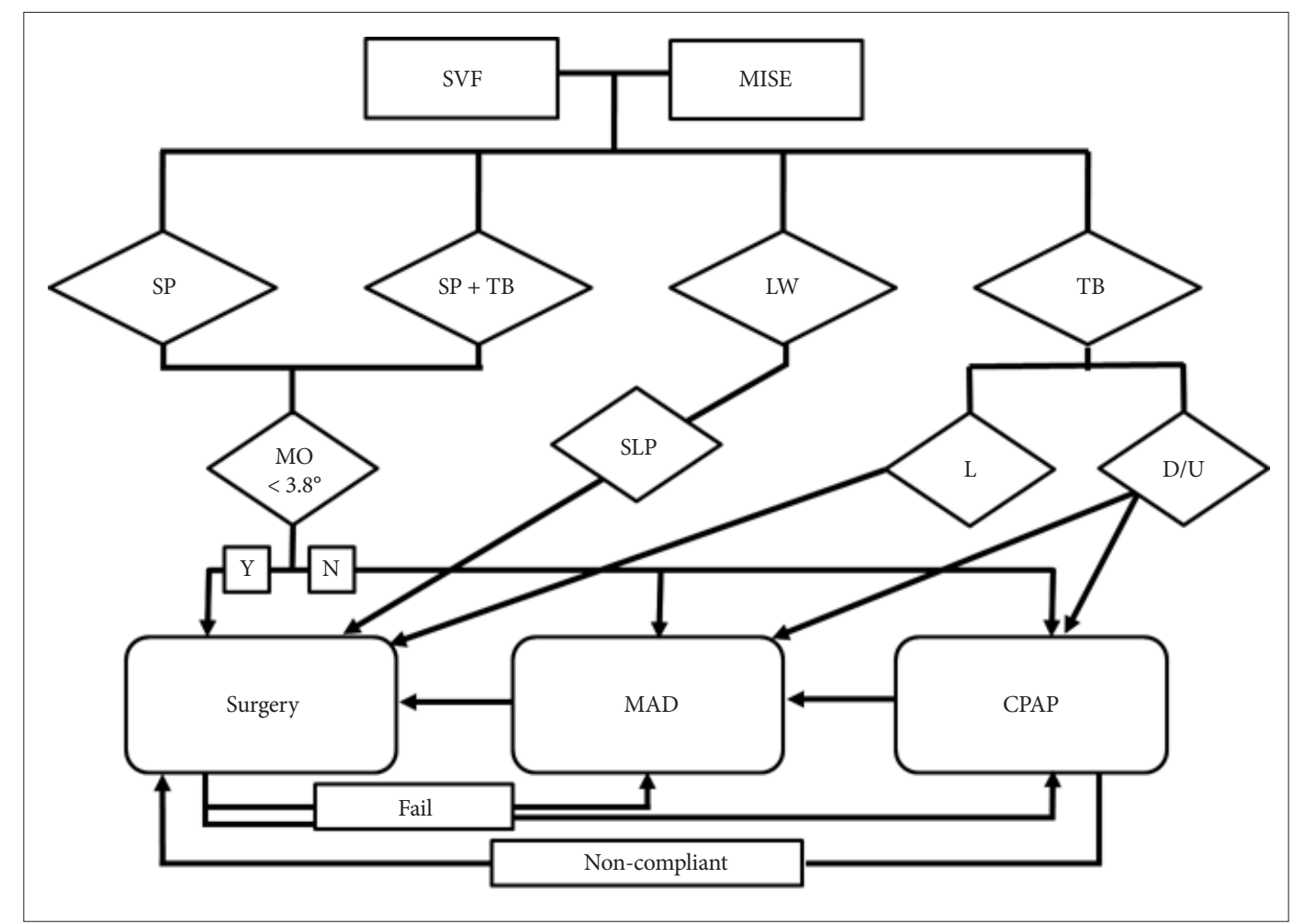

Fig. 6. Treatment algorism by dynamic upper airway evaluation. SVF: sleep video fluoroscopy, MISE: midazolam induced sleep endoscopy, SP: soft palate, TB: tongue base, LW: lateral wall, MO: mouth open, SLP: suspension lateral pharyngoplasty, L: lower, D: diffuse, U: upper, CPAP: continuous positive airway pressure, MAD: mandibular advancement device, Y: yes, N: no. 
snoring and sleep apnea patients. However, there are several limitations including the low sensitivity and specificity of the test, and different kind of instruments by different testers, which makes it difficult to compare the results between institutes.

\section{CONCLUSION}

Base of the study results, our department uses a treatment algorism shown in Fig. 6. First, we perform either drug induced sleep endoscopy or SVF to evaluate the obstruction level. If the obstruction site involves the SP, surgical treatment is indicated unless the mouth opening angle exceeds 3.8. Because the outcome of surgery is undesirable if the mouth angle is larger than $3.8^{\circ}$, CPAP treatment or MAD is considered. Tongue base obstruction is divided into 2 parts, upper tongue level and lower tongue level. Tongue base surgery is considered when the obstruction is found at the upper tongue level. In cases of upper or diffuse tongue level obstruction we do not perform surgery due to unsatisfactory outcomes, instead we prescribe nonsurgical treatment such as PAP or MAD. If the patient has an oropharyngeal lateral wall obstruction we consider both nonsurgical treatment and surgery to widen the lateral wall such as suspension lateral pharyngoplasty. Finally, PAP or MAD can be an alternative if surgery fails and when the patient's compliance to CPAP treatment is to low we consider surgery.

Unfortunately, a clearly defined modality for evaluating the airway obstruction level and also selecting a good surgical candidacy is not yet available in OSA patient. There is poor evidence to support the beneficial effect of certain upper airway evaluation methods on selected treatment outcome. With the help of these additional airway evaluation techniques, the underlying mechanics and pathogenesis of OSA is gradually coming emerging. However, the clinical importance of theses evaluation methods in daily practice is still insufficient. In fact, only conventional image methods are used in daily practice; nevertheless, the high techniques of upper airway evaluation can provide the information on the anatomical structure and spatial dynamics of the airway which enables physicians a proper treatment for OSA. The usage of these modalities often depends on the individual department's own experience and resources.

\section{Conflicts of Interest}

The authors have no financial conflicts of interest.

\section{REFERENCES}

1. Friedman M, Ibrahim H, Bass L. Clinical staging for sleep-disordered breathing. Otolaryngol Head Neck Surg 2002;127:13-21.

2. Friedman M, Ibrahim H, Joseph NJ. Staging of obstructive sleep apnea/hypopnea syndrome: a guide to appropriate treatment. Laryngoscope 2004;114:454-9.

3. Min YG, Jang YJ, Rhee CK, Kim CN, Hong SK. Correlation between anthropometric measurements of the oropharyngeal area and severity of apnea in patients with snoring and obstructive sleep apnea. Auris $\mathrm{Na}$ - sus Larynx 1997;24:399-403.

4. Riley R, Guilleminault C, Herran J, Powell N. Cephalometric analyses and flow-volume loops in obstructive sleep apnea patients. Sleep 1983; 6:303-11.

5. Liao YF, Chuang ML, Huang CS, Tsai YY. Upper airway and its surrounding structures in obese and nonobese patients with sleep-disordered breathing. Laryngoscope 2004;114:1052-9.

6. Battagel JM, Johal A, Kotecha B. A cephalometric comparison of subjects with snoring and obstructive sleep apnoea. Eur J Orthod 2000;22: 353-65.

7. Lee CH, Mo JH, Seo BS, Kim DY, Yoon IY, Kim JW. Mouth opening during sleep may be a critical predictor of surgical outcome after uvulopalatopharyngoplasty for obstructive sleep apnea. J Clin Sleep Med 2010; 6:157-62.

8. Otsuka R, Almeida FR, Lowe AA. The effects of oral appliance therapy on occlusal function in patients with obstructive sleep apnea: a shortterm prospective study. Am J Orthod Dentofacial Orthop 2007;131:176-83.

9. Robertson CJ. Dental and skeletal changes associated with long-term mandibular advancement. Sleep 2001;24:531-7.

10. Kim DK, Lee WH, Lee CH, Rhee CS, Kim JW. Interrater reliability of sleep videofluoroscopy for airway obstruction in obstructive sleep apnea. Laryngoscope 2014;124:1267-71.

11. Walsh JK, Katsantonis GP, Schweitzer PK, Verde JN, Muehlbach M. Somnofluoroscopy: cineradiographic observation of obstructive sleep apnea. Sleep 1985;8:294-7.

12. Lee CH, Hong SL, Rhee CS, Kim SW, Kim JW. Analysis of upper airway obstruction by sleep videofluoroscopy in obstructive sleep apnea: a large population-based study. Laryngoscope 2012;122:237-41.

13. Lee CH, Kim JW, Lee HJ, Yun PY, Kim DY, Seo BS, et al. An investigation of upper airway changes associated with mandibular advancement device using sleep videofluoroscopy in patients with obstructive sleep apnea. Arch Otolaryngol Head Neck Surg 2009;135:910-4.

14. Yucel A, Unlu M, Haktanir A, Acar M, Fidan F. Evaluation of the upper airway cross-sectional area changes in different degrees of severity of obstructive sleep apnea syndrome: cephalometric and dynamic CT study. AJNR Am J Neuroradiol 2005;26:2624-9.

15. Shepard JW Jr, Thawley SE. Evaluation of the upper airway by computerized tomography in patients undergoing uvulopalatopharyngoplasty for obstructive sleep apnea. Am Rev Respir Dis 1989;140:711-6.

16. Stuck BA, Maurer JT. Airway evaluation in obstructive sleep apnea. Sleep Med Rev 2008;12:411-36.

17. Moon IJ, Han DH, Kim JW, Rhee CS, Sung MW, Park JW, et al. Sleep magnetic resonance imaging as a new diagnostic method in obstructive sleep apnea syndrome. Laryngoscope 2010;120:2546-54.

18. Stuck BA, Köpke J, Hörmann K, Verse T, Eckert A, Bran G, et al. Volumetric tissue reduction in radiofrequency surgery of the tongue base. Otolaryngol Head Neck Surg 2005;132:132-5.

19. Huang Y, White DP, Malhotra A. The impact of anatomic manipulations on pharyngeal collapse: results from a computational model of the normal human upper airway. Chest 2005;128:1324-30.

20. Süslü AE, Oğretmenoğlu O, Onerci TM, Yücel OT. Comparison of two endoscopic examination methods, the Muller maneuver and fiberoptic pharyngoscopy during sleep, in patients with obstructive sleep apnea. Kulak Burun Bogaz Ihtis Derg 2006;16:200-4.

21. Woodson BT, Wooten MR. Comparison of upper-airway evaluations during wakefulness and sleep. Laryngoscope 1994;104:821-8.

22. Skatvedt O. Localization of site of obstruction in snorers and patients with obstructive sleep apnea syndrome: a comparison of fiberoptic nasopharyngoscopy and pressure measurements. Acta Otolaryngol 1993; 113:206-9.

23. Schoenberg SO, Floemer F, Kroeger H, Hoffmann A, Bock M, Knopp MV. Combined assessment of obstructive sleep apnea syndrome with dynamic MRI and parallel EEG registration: initial results. Invest Radiol 2000;35:267-76.

24. Petri N, Suadicani P, Wildschiødtz G, Bjørn-Jørgensen J. Predictive 
value of Müller maneuver, cephalometry and clinical features for the outcome of uvulopalatopharyngoplasty. Evaluation of predictive factors using discriminant analysis in 30 sleep apnea patients. Acta Otolaryngol 1994;114:565-71.

25. Doghramji K, Jabourian ZH, Pilla M, Farole A, Lindholm RN. Predictors of outcome for uvulopalatopharyngoplasty. Laryngoscope 1995; 105(3 Pt 1):311-4.

26. Katsantonis GP, Maas CS, Walsh JK. The predictive efficacy of the Müller maneuver in uvulopalatopharyngoplasty. Laryngoscope 1989;99 (7 Pt 1):677-80.

27. Lee CH, Mo JH, Kim BJ, Kong IG, Yoon IY, Chung S, et al. Evaluation of soft palate changes using sleep videofluoroscopy in patients with obstructive sleep apnea. Arch Otolaryngol Head Neck Surg 2009;135: $168-72$.

28. Sadaoka T, Kakitsuba N, Fujiwara Y, Kanai R, Takahashi H. The value of sleep nasendoscopy in the evaluation of patients with suspected sleep-related breathing disorders. Clin Otolaryngol Allied Sci 1996;21: 485-9.

29. Marais J. The value of sedation nasendoscopy: a comparison between snoring and non-snoring patients. Clin Otolaryngol Allied Sci 1998;23: 74-6.

30. Berry S, Roblin G, Williams A, Watkins A, Whittet HB. Validity of sleep nasendoscopy in the investigation of sleep related breathing disorders. Laryngoscope 2005;115:538-40.

31. Steinhart H, Kuhn-Lohmann JC, Gewalt K, Constantinidis J, Mertzlufft F, Petak M, et al. [Pharyngolaryngoscopic findings in patients with obstructive sleep apnea syndrome and primary snoring]. HNO
2000;48:917-21.

32. Camilleri AE, Ramamurthy L, Jones PH. Sleep nasendoscopy: what benefit to the management of snorers? J Laryngol Otol 1995;109:1163-5.

33. Kezirian EJ, Hohenhorst W, de Vries N. Drug-induced sleep endoscopy: the VOTE classification. Eur Arch Otorhinolaryngol 2011;268:1233-6.

34. Tvinnereim M, Mitic S, Hansen RK. Plasma radiofrequency preceded by pressure recording enhances success for treating sleep-related breathing disorders. Laryngoscope 2007;117:731-6.

35. Lee CH, Won TB, Cha W, Yoon IY, Chung S, Kim JW. Obstructive site localization using multisensor manometry versus the Friedman staging system in obstructive sleep apnea. Eur Arch Otorhinolaryngol 2008;265: 171-7.

36. Faber CE, Grymer L, Norregaard O, Hilberg O. Flextube reflectometry for localization of upper airway narrowing--a preliminary study in models and awake subjects. Respir Med 2001;95:631-8.

37. Herzog M, Schmidt A, Bremert T, Herzog B, Hosemann W, Kaftan H. Analysed snoring sounds correlate to obstructive sleep disordered breathing. Eur Arch Otorhinolaryngol 2008;265:105-13.

38. Agrawal S, Stone P, McGuinness K, Morris J, Camilleri AE. Sound frequency analysis and the site of snoring in natural and induced sleep. Clin Otolaryngol Allied Sci 2002;27:162-6.

39. Xu H, Huang W, Yu L, Chen L. Sound spectral analysis of snoring sound and site of obstruction in obstructive sleep apnea syndrome. Acta Otolaryngol 2010;130:1175-9.

40. Won TB, Kim SY, Lee WH, Han DH, Kim DY, Kim JW, et al. Acoustic characteristics of snoring according to obstruction site determined by sleep videofluoroscopy. Acta Otolaryngol 2012;132 Suppl 1:S13-20. 